\title{
Une biographie de la lanterne à « réverbères » : gestion collective de l'innovation, script technique et réticulation spatiale de l'artifice
}

\author{
A history of the street light à réverbères: collective management of the \\ innovation, technical script and space reticulation
}

\author{
Benjamin Bothereau ${ }^{1}$ \\ ${ }^{1}$ Centre Alexandre Koyré, EHESS, Paris, France, bbothere@gmail.com
}

RÉSUMÉ. La lanterne " à réverbères » aussi appelée " optique » est une version améliorée de la lanterne ordinaire qui optimise l'intensité lumineuse en utilisant des artifices, les réflecteurs métalliques concaves (réverbères), afin de diriger le flux lumineux. Si l'historiographie s'est focalisée sur la lampe d'Argand, nous avons ainsi construit notre étude autour d'un élément technique moins noble, peu valorisé, le réflecteur métallique. Partant de l'artifice vers l'objet en action de rue, l'objectif de cet biographie d'innovation est de mettre en valeur les modes d'existence de la lanterne, en particulier sa genèse technique et sa technophanie, afin de redonner l'épaisseur sociale et politique que cet objet, en apparence trivial et banal, a pu revêtir et sa participation à la culture technique.

ABSTRACT. The street light à réverbères, also known as the "optical" street light, is an improved version of the ordinary street light which optimizes the luminous intensity of the lantern by using concave metal reflectors to direct the beam of light where needed. Although history chooses to focus on the Argand lamp, this article deals with a far less dignified and valued technical element: the reflector. From the technical scheme to its use, this paper presents the history of this lighting innovation, and seeks to shed light upon the multi-layered and entwined nature of this seemingly trivial and banal artefact.

MOTS-CLÉS. Biographie d'objet technique, innovation d'éclairage, technophanie, imaginaire technique, police, territoire urbain, abstraction géométrique, rationalisation, réticulation spatiale, l'objet et son milieu.

KEYWORDS. Biography of a technical objet, lighting innovation, technophanie, technical imaginary, police, urban territory, geometric abstraction, rationalization, space reticulation, the object and its milieu.

Si l'approche micro-historique a parfois été définie comme une histoire « au ras du sol » [REV 96], nous avons pour objectif de construire une histoire de la lanterne « au ras de l'objet ». L'objet technique étant une construction sociale et culturelle, nous souhaitons lire les imaginaires qui ont formé la lanterne comme autant de couches géologiques, et les transformer en matière première pour les analyser [MUS 15]. Nous proposons ainsi une biographie de la lanterne à réverbères, faisant écho aux contributions réunies de The Social life of Things [APP 86] pour une biographie culturelle et sociale des objets, mais en plaçant cette fois-ci l'analyse technique de l'objet en premier plan, servant de fil conducteur entre nos parties. Fortement influencé par l'anthropologie sociale et son recentrage sur les pratiques (practical turn) [BEN 12], le genre biographique s'étend aux artefacts, instruments, machines et systèmes, générant alors un «tournant vers les choses » (thingly turn) [VER 05] pour l'étude des sciences et des techniques à la fin du $\mathrm{XX}^{\mathrm{e}}$ siècle.

L'émergence de la nouvelle technique des réverbères pour l'éclairage public au XVIIIe s. est intrinsèquement accompagnée de discours, d'images, de mythes, de récits, qui structurent son imaginaire. C'est à travers ce fonds culturel que nous souhaitons appréhender notre objet. La biographie s'offre alors comme une ressource précieuse : cette méthode centrée sur l'objet conduit à une perception de sa technicité et de ses valeurs associées à des niveaux différents mais noués et enchevêtrés. Nous avons donc opté, dans la construction de notre biographie, pour une succession de prismes qui révèlent l'objet- dans son schème, dans sa valeur fonctionnelle ou symbolique. Nous nous affranchirons de la distinction objet/sujet et de la partition entre nature et culture pour traiter 
notre entité technique «lanterne » comme acteur. C'est en tournant le dos à cette distinction ontologique que la théorie de l'acteur-réseau (ANT) [AKR 06] [LAT 91] a pu traiter l'objet et définir l'acteur (humain ou non humain) non par sa nature, mais par ses performances et par ses effets sur les autres acteurs auxquels il est lié. C'est également dans cette dynamique que Gilbert Simondon, dès 1958, a pu questionner les modes d'existence des objets techniques et les penser en devenir, dans un processus d'individuation. L'objet advient à l'existence dans un processus de «concrétisation », il s'invente en intégrant dans son fonctionnement les effets de son opération sur lui-même et sur le milieu associé [SIM 89]. Comment mettre en évidence le caractère réflexif et agissant de la culture qui met en relation la technique et le monde environnant ? L'approche biographique de notre objet va nous permettre d'apporter notre modeste contribution au développement de la culture technique en montrant d'une part les efforts humains précédents la génèse technique [GAR 03] de la nouvelle lanterne et d'autre part les multiples régimes d'appropriation de la nouvelle technique, faisant entrer notre objet dans le monde des significations à travers sa technophanie :

«La « technophanie » désigne dans un objet la résonance d'un ensemble d'objets auquel il appartient et avec lesquels il fonctionne. La technophanie d'un objet technique correspond à la manière dont cet objet se fait le «symbole » de l'ensemble auquel il appartient » [BEAU 13].

Nous souhaitons donc approcher ce «symbole» dans cet article, en interrogeant les résonances de l'objet technique dans son «ensemble », et inversement, grâce à la méthode biographique. Si l'Etat absolutiste a la volonté de soumettre la rue à son ordre via des procédures pour quadriller, contrôler, mesurer et discipliner les individus [FOU 75], l'implantation de l'éclairage public matérialise cette intention. Ce mode de la lanterne politique va être ainsi défini par le double imaginaire de l'administration monarchique et du contrôle policier. Les binômes de l'éclairage et de la police sont connus $^{1}$ : d'une part la fondation de la lieutenance -le concours « Sartine » - pour la captation de savoirs techniques de l'éclairage, d'autre part, l'accaparement de compétences et la centralisation de la gouvernance de l'illumination sous l'égide de cette même lieutenance. Dans cet article, nous avons voulu dépasser ces premiers résultats, et aller également au-delà de la propagande monarchique jouant, à peu de frais, sur la naissance conjointe de l'institution d'éclairage et de la lieutenance de police parisienne (1667). Fidèle à une histoire biographique « au ras de l'objet », nous avons souhaité identifier de potentielles résonances de la culture d'agir de la lieutenance de police parisienne dans la matérialité de l'objet technique.

Nous nous intéresserons ainsi tout d'abord au cadre du processus d'innovation de la lanterne à réverbères. Ce premier moment biographique se focalise sur le nouveau script de compétences [AKR 91]dans le dispositif technique par les inventeurs, l'Académie des sciences et la lieutenance de police parisienne. Cette descente dans le schème technique de l'objet nous apparait indispensable pour saisir l'imaginaire technique de la lanterne et la place - que nous montrerons essentielle - qu'y tient son artifice (le réverbère). Nous interrogeons alors la technicité de l'objet d'éclairage en l'extrayant dans un premier temps de son milieu « naturel », c'est à dire en la prenant ex situ de son action de rue. Nous montrerons en particulier que le réflecteur métallique « réverbère » devient la matérialisation des processus de maitrise et de rationalisation des rayons lumineux.

\footnotetext{
${ }^{1}$ Auguste-Philippe Herlaut, "L'éclairage des rues à Paris à la fin du XVIle et au XVIIle siècles ", Mémoires de la Société de I'Histoire de Paris et de l'île de France, vol. XLIII, 1916 ; Auguste-Philippe Herlaut, L'éclairage de Paris à l'époque révolutionnaire, Paris, Mellotée, 1933. Pour une étude de l'illumination comme instrument de contrôle policier, voir également Catherine Denys, Police et sécurité au XVIIle siècle dans les villes de la frontière francobelge ,Paris, L'Harmattan ; Jean-Luc Laffont, Policer la ville. Toulouse, capitale provinciale au siècle des Lumières, thèse de doctorat en histoire, université de Toulouse II Le Mirail, 1997.
} 
Interroger le sens de la technicité du nouvel objet n'est pas suffisant, il faut également étudier la lanterne revisitée «en action de rue». Ainsi, l'artefact technique ne peut être pensé sans la ville : plus que son écrin, l'espace urbain est en interaction avec lui, la rue étant l'objet complémentaire qui assure sa «réticulation spatiale»-au sens de Simondon [SIM 60]. Nous avons donc construit le deuxième moment biographique pour comprendre l'intégration de l'objet à la ville non réduite à une conquête des éléments (la flamme, les rayons lumineux) par une science raisonnée mais matérialisant une vision maitrisée de l'espace urbain grâce à la technique. La lanterne publique rationalisée rayonne donc au-delà de ses limites matérielles, au moyen d'un prolongement de sa technicité et de son imaginaire à l'ensemble plus complexe de l'objet en action de rue.

\section{L’objet. Matérialisation du nouveau script de compétences dans la lanterne publique}

Si nous débutons notre biographie de la lanterne par le prisme du Concours d'éclairage (17631766), coproduction de l'Académie des sciences et de la lieutenance de police de Paris, nous ne souhaitons en aucun cas alimenter une hagiographie des inventeurs de la lanterne optique qui tronquerait le mode d'existence de l'innovation d'éclairage. Si l'on regarde d'ailleurs de plus près les dispositifs techniques lauréats, on s'aperçoit que les récompenses sont attribuées à des solutions techniques contradictoires. Le partage du prix entre les trois inventeurs -Bailly, de Chateaublanc et Leroy- aux méthodes divergentes laisse apercevoir la complexité de la conception technique telle qu'elle est vue par l'administration technicienne : pensée combinatoire, processus cumulatifs de la connaissance, soit une gestion collective de l'invention et du progrès ${ }^{2}$, loin de l'héroïsation de l'inventeur Bourgeois de Chateaublanc comme «père de la lanterne à réverbères » ou de la célébration du génie inventif à travers la remise de la médaille d'or à Lavoisier le 9 avril 1766 pour son mémoire sur l'illumination, reprises toutes les deux dans l'historiographie. Ce qui nous intéresse ici n'est pas l'individu mais bien la production de l'imaginaire de l'artifice (le réverbère) en tant que réponse rationalisée au problème d'éclairage.

\subsection{Une mise en problème comme discours du pouvoir : optimiser les trois paramètres «clarté», «service» et «économie»}

Le programme du Prix est une réduction de l'enjeu de l'éclairage public dans les termes d'un problème. La mise en problème opère d'une réduction (au sens d'Hélène Vérin [DUB 08]) : la grande complexité du réel nocturne fait place à des catégories. La construction du problème est une réduction des éléments à considérer : «sur la meilleure manière d'éclairer [...] en combinant ensemble, le mieux qu'il sera possible, la clarté, la facilité du service et l'économie ». Nous proposons de lire ce programme au prisme des travaux d'Hélène Vérin, notamment La gloire des ingénieurs qui s'intéresse à la poursuite non du vrai mais du «meilleur possible». En ce sens, il nous semble que le programme du prix d'éclairage se rapproche des enjeux d'une ingénierie de l'éclairage, cherchant l'optimisation entre les trois éléments «clarté », « service » et « économie » et non pas la vérité savante des lois de l'éclairage extérieur. La solution technique d'éclairage doit donc consister à mettre en balance des avantages hétérogènes, voire a priori contradictoires tension entre la tentation de multiplier les sources lumineuses (clarté) et la demande de moindre consommation en combustible (économie) - et à trouver une solution d'équilibre.

Arrêter ces trois paramètres, c'est également circonscrire les épreuves pour donner un sens à la comparaison entre les systèmes proposés par les concurrents. De plus la circonscription à ces trois éléments et la nature de ces derniers est révélatrice des éléments jugés pertinents pour le programme d'éclairage. C'est donc bien une exigence de combinaison des trois paramètres, une pondération des

\footnotetext{
${ }^{2}$ Pour une étude détaillée des rapports de force autour des " valeurs » de la lanterne et sur une " controverse du réverbère ", voir [BOT 18].
} 
avantages qui est voulue comme le montre le rejet de la pièce $n^{\circ} 11$ par la commission ${ }^{3}$ : il s'agit d'un mémoire de catoptrique de $\mathrm{M}$. Genty, professeur à Orléans qui recherche la forme la plus avantageuse à donner aux réverbères en calculant les quantités de lumière réfléchie. Pour les commissaires, «c'est une simple théorie, il n'est pas question dans son mémoire des autres conditions du problème posé ». Ils écartent donc ce mémoire, actant que « cet ouvrage n'est pas dans le cas de concourir au Prix ».

Le programme du Prix est un cahier des charges de l'objet technique, hybride dans son descriptif entre les devis de fabrication de lanternes et les baux du service d'éclairage. Si l'énoncé du Concours mentionne une seule fois la notion de performance en cherchant la " clarté », le détail du cahier des charges laisse transparaitre la vraie valeur du dispositif technique recherché. Sur les sept points auxquels doit répondre la solution d'éclairage, seul le point 4 concerne la disposition des lanternes pour «augmenter la lumière ». Si ce point précise de «diminuer les ombres » c'est que c'est moins l'augmentation de la luminosité que l'homogénéité lumineuse qui est à la clé. Les finalités du dispositif technique sont en revanche clairement établies dans les points 1, 2, 6 et 7. Le premier et le deuxième point s'intéressent au coût et à la durabilité du dispositif en demandant une étude sur la nature du combustible pour «diminuer ses inconvénients et son prix», rendre la flamme «plus capable de résister soit au vent, soit à l'humidité de l'air, soit à la gelée » et un travail sur la nature de la mèche pour «éclairer toujours également pendant plusieurs heures». Les sixième et septième points combinent une demande en solidité et en fonctionnalité du dispositif avec une recherche des « suspensions les plus solides et les plus commodes» et des «constructions et dispositions les plus favorables pour la solidité et la facilité du service ». La nouvelle valeur du système d'éclairage- « la clarté, la facilité du service et l'économie » combinés- s'ancre donc bien dans la dynamique de recherche des «moyens d'économie» [VER 93] qu'ils offrent à la nation ou à la société ${ }^{4}$. La question financière est également primordiale ici car l'éclairage public est une source de dépenses, enflant jusqu'à devenir le premier poste de dépense de la police parisienne dans les années 1780. Comme le montre Hélène Vérin, l'exigence du bien public dans la rhétorique du pouvoir, va réévaluer les avantages des objets techniques : plus que la performance, ce sont le coût des réalisations, la solidité des objets, leur fonctionnalité et leur durée qui intéressent alors l'économie du pays. La presse d'annonces techniques se fait le relai de cette politique technicienne dans un article de 1765 consacré à la présentation du concours :

«L'intention des magistrats a même été de proposer un prix à celui qui fournirait sur cet objet quelque invention utile [...] Ces lampes produisent un effet bien supérieur à nos lanternes à l'égard du choix dans les formes tentées; il faut d'abord peser les frais, la promptitude du service, l'avantage qui en résulte, après quoi l'on pourra décider celle à laquelle on doit donner la préférence. » (L'Avant-Coureur, n' 8,1765$)$

Conscient que la seule performance n'est pas l'objectif, le journal ne réduit par «l'invention utile » à son « effet » mais bien aux avantages qu'il procure.

En août 1763, au moment de construire le programme du concours, l'innovation du réverbère est déjà connue à la fois de l'Académie des Sciences - qui a déjà évalué les lanternes à réverbères de Chateaublanc - et du lieutenant de police puisque Chateaublanc le prend comme caution dans son privilège de manufacture royale de lanternes. L'énoncé atteste d'ailleurs de ce travail préalable en précisant «on n'a point encore suffisamment étudié... ». Ce qui intéresse l'administration technicienne se glisse dans la partie de l'intitulé du concours souvent oubliée dans l'historiographie :

\footnotetext{
${ }^{3}$ N90. Rapport des soumissions du second tour, Archives du Musée des Arts et Métiers, Réserves de Saint Denis.

${ }^{4}$ En parallèle, l'exemple de Jacques Vaucanson permet d'illustrer cette administration technicienne qui pense la technique en terme d'intérêt public et donc de moyens d'économie dans l'industrie de la soie.
} 
« combiner ensemble, le mieux qu'il sera possible » ${ }^{5}$. On attend donc moins du participant une invention qui vise la performance lumineuse qu'une capacité à combiner les savoirs, une méthode pour rationaliser les valeurs du dispositif technique d'éclairage. Si un point concerne effectivement exclusivement les «nouveaux » réflecteurs métalliques, il a l'intérêt de nous renseigner sur la position des commanditaires vis à vis de cette innovation. La question très ouverte - « s'il faut y mettre des réverbères, de quelle figure ils doivent être, et comment on doit les appliquer »- n'oblige donc pas le participant à composer la lanterne avec des réflecteurs mais le contraint à argumenter de son usage ou non. Orienter l'invention dans ce cas n'est pas imposer l'utilisation de l'innovation (réflecteur ou lampe à huile) mais demander au participant d'envisager et de combiner tous les possibles. C'est la matérialisation de la pondération de ces avantages et de l'équation de la lanterne que nous étudions dans ce qui suit.

\subsection{Le réverbère : l'artifice pour rationaliser les rayons lumineux}

Les deux premiers modèles techniques de lanternes (sans réverbères) installés en amont du prix d'éclairage dans les rues parisiennes sont les lanternes à seau puis les lanternes à cul-de-lampe, déclinaisons de la version précédente, à partir des années 1730. Ces lanternes sont de forme octogonale, vitrées au plomb par huit pans totalisant vingt-quatre pièces de verre. Cette multiplication des interfaces et l'épaisseur des plombs - « de cinq lignes de large, compris le cœur qui sera d'une ligne $»^{6}$ - obstruent la lumière générée par la chandelle. De plus, une platine et deux bobèches génèrent un «embarras » $\mathrm{du}$ fond de la lanterne et un deuxième obstacle pour le rayonnement lumineux. Cette combinaison des cônes d'ombre produit donc des asymétries de l'intensité lumineuse sur le pavé. Les descriptions des ombres mouvantes de l'ancien parc d'éclairage se retrouvent ainsi indifféremment dans la littérature générale -avec Louis-Sébastien Mercier - ou technique, via le traité du vitrier Le Vieil [VIE 74]:

«Autrefois, huit mille lanternes avec des chandelles mal posées, que le vent éteignait ou faisait couler, éclairaient mal, et ne donnaient qu'une lueur pâle, vacillante, incertaine, entrecoupée d'ombres mobiles et dangereuse. ».

«Les chandelles entretenaient un jour louche et les plombs formaient sur le pavé de grandes ombres, d'autant plus multipliées qu'il y avait plus de lanternes. »

Le champ d'action des lanternes est également inégal. Ainsi, le commissaire Delamare décrit au lieutenant de Police de la Reynie les limites de l'efficacité des lanternes ordinaires en remettant en cause leur champ d'action sur le pavé :

«L'expérience fait voir que toute cette grande quantité de lanternes qu'on a mises d'abord et l'augmentation qu'on en a faite depuis ne produit pas un si bon effet que l'on en attendait, la clarté n'en étant pas de beaucoup augmentée et tout l'avantage qu'on en tire, c'est de faire voir des feux semblables à ceux qui sont sur les ports et les cotes de la mer pour marquer et non pas pour éclairer les chemins. $»^{7}$

L'analogie avec le phare employée par le commissaire est particulièrement révélatrice : la lanterne est visible car elle est lumineuse mais son champ d'action local - au sens de pouvoir éclairant - est limité par l'existence d'un cône d'ombre généré sous l'espace de suspension.

\footnotetext{
${ }^{5}$ Archives de l'Académie des Sciences, pochette de séance, « Concours », 1763

${ }^{6}$ BNF, Département des manuscrits, Français 21684 fol 334335 devis pour la fabrication et l'entretien des lanternes publiques des quartiers du Louvre, du Palais Royal, de Montmartre et de Saint Eustache du 6 avril 1730.
}

${ }^{7}$ BNF Msfr 21684. 


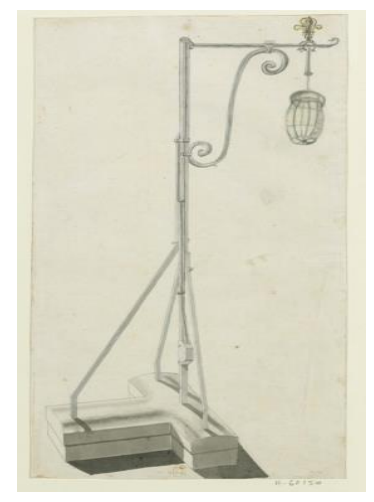

Figure 1. Lanterne «à cul de lampe » (sans réverbères), Cours -la- Reine, $1729^{\circ}$

En complément de l'enjeu d'économie, l'innovation d'éclairage doit ainsi convertir ce maillage de repères lumineux ponctuels - décrit par Delamare - en un système qui tend à éclairer l'espace urbain de façon homogène et continue. Ces intentions sont concentrées dans l'artifice du réverbère qui est présenté comme le vecteur de la suppression des ombres en optimisant et en orientant les rayons vers la surface utile de la rue - le pavé. Le réflecteur métallique « réverbère » devient alors la matérialisation des processus de maitrise et de rationalisation des rayons lumineux. Le discours du mécanicien-inventeur Chateaublanc inscrit l'utilisation de l'artefact réverbère comme moyen pour contrer la propagation naturelle - contre la liberté des rayons lumineux « de s'échapper selon leur direction naturelle » et « de se perdre dans le vague de l'air» - et optimiser le parcours lumineux contre «une certaine quantité qui se porte dans des endroits où ils sont inutiles ». Le réflecteur matérialise ainsi la maitrise et l'action de l'inventeur sur la lumière : "on a adapté de petits réverbères destinés à renvoyer la lumière de côté où l'on veut la diriger, et afin de la faire parvenir à de plus longues distances ».

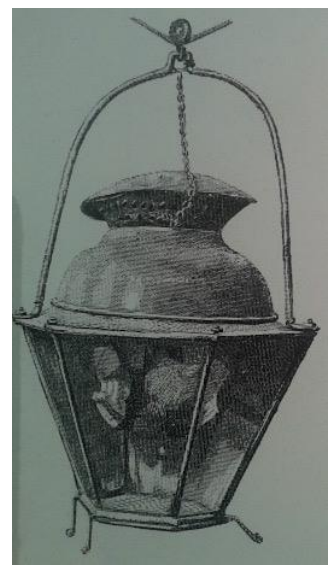

Figure 2. La lanterne à réverbères

Répondre au programme d'éclairage, c'est balancer les avantages de clarté et d'économie. Au lieu de travailler sur l'augmentation des sources -multiplication des chandelles ou des mèches à huile- et de tomber sur une impasse, l'inventeur doit introduire dans le système un artifice - le réverbère- pour «conduire les effets» de la lumière. Cette démarche se rapproche des intentions des ingénieurs étudiées par Hélène Vérin qui veulent « conduire les effets de leur intention » à travers la simulation, procédure qui leur permet de réduire le problème à des rapports entre intentions et données, entre intentions et effets.

\footnotetext{
${ }^{8}$ Lanterne « à cul de lampe ». Robert de Cotte, Différents plans relatifs à l'aménagement du Cours -la- Reine, 1729, Bibliothèque Nationale de France, Paris [FOL-VA-278 $(F, 3)]$.
} 
« Si l'on est touché de la dépense et qu'en conservant la clarté le plus qu'il sera possible, on veuille la combiner avec l'économie, les réverbères qui augmentent la force de la lumière et la projettent à de plus grandes distances, sont la seule ressource qui reste pour concilier l'une et l'autre. »"

A la performance lumineuse - celle que peut juger facilement le public et qui crée l'émerveillement de ce dernier - Lavoisier insiste ainsi sur «l'objet d'économie qui pèse fort dans l'équation de la lanterne». Pour l'auteur, travailler sur la puissance des sources lumineuses ne suffit pas, il souhaite dans un premier temps approcher l'idéal du réverbère, c'est à dire parfaire le réflecteur pour optimiser le trajet optique, maitriser les rayons lumineux pour générer des possibilités d'économiser le combustible. L'utilisation du support papier et de la figuration permet alors à l'inventeur de géométriser l'espace de la lanterne, de faire converger les propriétés mathématiques et les effets techniques du réverbère, c'est à dire de formaliser les intentions. Le dessin permet d'anticiper les effets des formes possibles : c'est bien la lumière qui se doit d'être maitrisée par la figuration :

«Il s'agit d'examiner les ressources que nous fournit la Physique, d'employer les moyens qu'elle nous donne pour augmenter l'effet des lumières, pour en multiplier l'image. Elle peut nous procurer tous ces avantages, nous le savons, pourquoi ne chercherions-nous pas à en profiter ? ${ }^{10}$

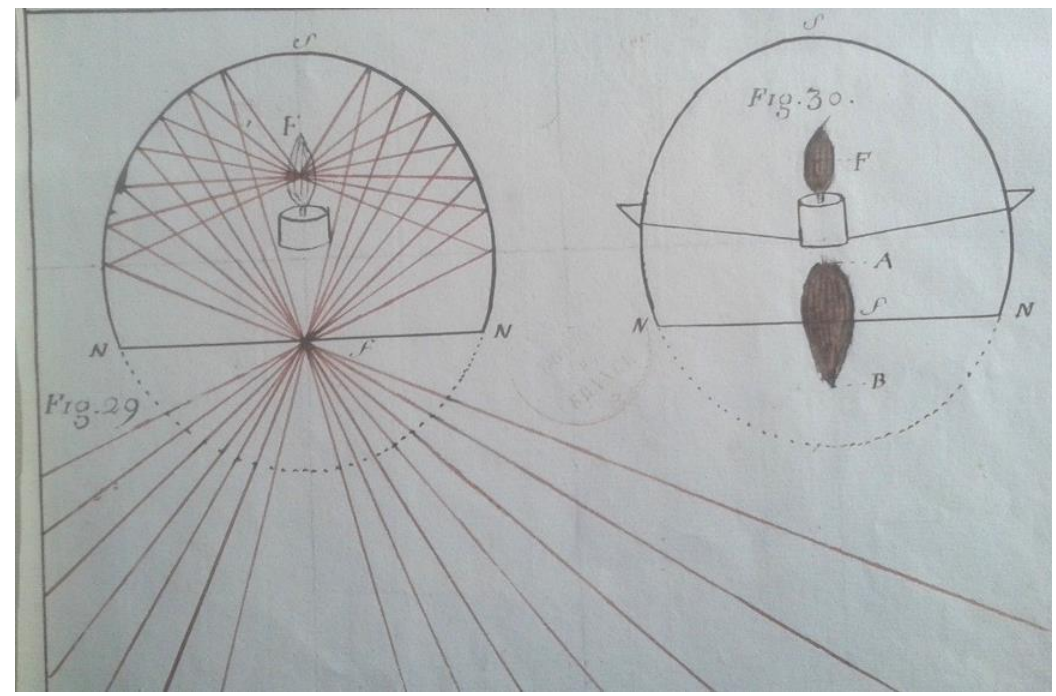

Figure 3. Simuler les effets du sphéroïde elliptique, mémoire de Lavoisier, 1765

\section{L'objet et son milieu. La lanterne à la ville.}

«La réticulation spatiale consiste en ce fait que la technicité ne peut être contenue en un seul objet ; un objet n'est technique que s'il opère en relation avec d'autres objets, dans un réseau où il prend la signification d'un point-clef ; en lui-même et comme objet, il ne possède que des caractères virtuels de technicité qui s'actualisent dans le rapport actif à l'ensemble d'un système. La technicité est une caractéristique de l'ensemble fonctionnel qui couvre le monde et dans lequel l'objet prend une signification, joue un rôle avec d'autres objets.» [SIM 60] ;

\footnotetext{
${ }^{9}$ Mémoire de M. Bailly, 1765, Archives de l'Académie des sciences. Mémoire lauréat du prix académique d'éclairage (17631766).
}

${ }^{10}$ Mémoire de Lavoisier, 1765, Archives de l'Académie des sciences. Mémoire pour le prix académique d'éclairage (1763-1766). 
Notre deuxième moment biographique nous permet, après la phase de conception, d'analyser un nouveau mode d'existence de la lanterne à réverbères, où cette dernière accomplit sa technophanie, en se faisant le « symbole » de l'ensemble - ici le système « rue »- auquel elle appartient.Dans un contexte de transformations physiques et d'embellissement de la capitale ${ }^{11}$ portés par des percées et des plans d'alignement, nous étudierons l'intégration de la lanterne à la ville. De quelle façon les discours de rationalisation de l'artifice du réverbère relevés précédemment se prolongent-ils dans l'inscription spatiale de la lanterne? Quels sont les mécanismes de la traduction et de la transposition du script de compétences de notre objet technique au milieu ? Comment et par qui sont-ils légitimés ?

\subsection{La lanterne intègre les signes visuels de l'interventionnisme de l'Etat dans la rationalisation territoriale}

Un territoire urbain nocturne qui tend à devenir rationalisé et homogénéisé requiert les mêmes qualités de l'outil de sa réforme, la lanterne. Ainsi, pour prétendre à un éclairage homogène de la ville, un maillage régulier et une disposition à l'identique est nécessaire sur tout le tissu urbain. L'édit de 1667 ne fixe pas la distance inter-lanternes en ce sens qu'elle n'ordonne que la densification de l'éclairage des rues et laisse donc la place à des installations non normées de lumières complémentaires entre les lanternes précédemment installées, dans la pratique une à chaque extrémité de rue, et pour les rues considérées «longues»-sans indication de mesure minimale- une lanterne supplémentaire au milieu de celles-ci «dans les endroits les plus commodes $»^{12}$. C'est véritablement l'édit de 1697 qui va normaliser la configuration de l'éclairage public en ordonnant que les lanternes soient «posées de cinq à six toises de distance l'une de l'autre, de manière qu'elles fussent directement au milieu des rues » $(9 \mathrm{~m} 70 \mathrm{~cm}$ à $11 \mathrm{~m} 70 \mathrm{~cm}$ soit 10 à 12 m environ). L'incertitude sur la distance ( 1 toise) soit $20 \%$ de précision sur la distance de base 5 toises peut étonner dans cette dynamique de normalisation et de rationalisation de la rue. Cependant, il ne faut pas perdre de vue que l'édit de 1697 correspond à la deuxième étape de mise en place de l'éclairage et que nous sommes toujours dans une phase d'expérimentation.

Comment la lanterne participe-t-elle de la mise en ordre de l'espace urbain ? L'ordonnancement de la rue par le marqueur «lanterne », la régularité souhaitée de l'interdistance des lanternes et le traitement uniforme de l'ensemble des rues, participent plus largement d'une volonté de mise en ordre urbanistique. Loin du goût architectural et stylistique, une volonté d'ordonnancement et de symétrie transparait dans les écrits policiers. Leclerc du Brillet rédige le chapitre "Voierie » du quatrième volume du Traité de la police dans les mêmes années (1720-1730) est en contact avec le procureur général Joly de Fleury. Il étudie le modèle romain du bâti et montre les bienfaits de la régularité des rues dans son titre III «De la largeur et de l'alignement des rues », éléments qui rentrent pleinement dans l'équation de l'éclairage -fixation, portée de la lumière, hauteur, distance entre lanternes en fonction de la largeur des rues, etc. Pour du Brillet, la régularité du tracé des rues est synonyme de «progrès » et conditionne la « grandeur, beauté et magnificence » de Paris.

\subsubsection{Le Cours-la-Reine, un « laboratoire » idéal de l'éclairage ?}

La version manuscrite du traité de Police, (collection Delamare continuée par Le Cler Du Brillet, fol 98) donne l'analyse de cette régularité au prisme de l'institution : " cet établissement sera autant pour l'ornement de cette ville que pour la sûreté et la commodité du Grand Chemin ». La question

\footnotetext{
${ }^{11}$ Pour une étude complète des transformations physiques de la capitale (1750-1815), le boom du secteur de la construction, deuxième secteur économique après l'agriculture, le contrôle de la construction privée (plan d'alignements, etc.) et le rôle essentiel d'intermédiaire des corporations du monde du bâtiment dans les contrôles de qualité des constructions, avant et après la Révolution, voir [РОТ 09a], [РОТ 09b].

${ }^{12}$ MSF 21684 fol 153.
} 
de sûreté et de commodité n'est mentionnée qu'une fois, sans approfondissement. Elle fait écho à l'arrêt du 21 décembre $1728^{13}$ sur l'établissement des lanternes du Cours qui mentionne ces deux notions : «Le roi étant informé du danger qu'il y a pour les voitures et pour les gens de pied de passer dans les nuits obscures sur la chaussé du Cours la Reine [...] n'y ayant ni parapet ni défenses du côté de la rivière ni du côté du fossé où il est tombé plusieurs personnes et plusieurs voitures depuis quelques années ». Les lanternes contribueront alors à rendre cette route « sûre » et «à éviter les accidents ». En revanche, le traité est beaucoup plus exhaustif sur les effets de la rationalisation de l'emplacement des lanternes. La question esthétique et le souci d'ordonnancement, de police de la ville, sont associés à la régularité du dispositif, ici sur un échantillon de soixante lanternes :

«L'égalité de distance entre ce grand nombre de lanternes et l'uniformité de la hauteur à laquelle elles sont suspendues et la lumière qu'elles réfléchissent dans le canal de la rivière [...]forment un coup d'œil qui satisfait infiniment et qui annonce une ville opulente et bien policée, en un mot la capitale du royaume. »

Le binôme est donc indissociable. Pierre Patte, un architecte qui participe au concours d'éclairage décrit ainsi Paris : "des amas de maisons distribuées sans ordre, sans entente d'un plan total convenablement raisonné» [PAT 69, p.5]. Pierre Patte propose la destruction totale du bâti de la Cité, souhaitant établir un quadrillage de rues droites : «A l'exception de Notre-Dame qui resteroit paroisse de la Cité, \& du bâtiment des Enfans-Trouvés, il n'y auroit rien à épargner dans ce quartier ». Il est présenté comme un des plus fervents propagandistes des théories aéristes et d'aménagement urbain. Certains auteurs ont montré que les projets d'embellissements se multiplient au siècle des Lumières - alignement des rues étroites et tortueuses, percées monumentales, dégagement des quais - mais demeurent inachevés, partiels ou sans cohérence [CAR 09], en se heurtant aux coûts tout d'abord mais aussi à la force des habitudes constructives, à la faiblesse du personnel de la surveillance de la voirie et des bâtiments, et surtout à l'insuffisance de l'arsenal législatif et règlementaire qui ne permet pas vraiment de procéder à des expropriations efficaces. Il nous semble que l'idéal de normalisation spatiale peut au moins se réaliser dans l'éclairage parisien.

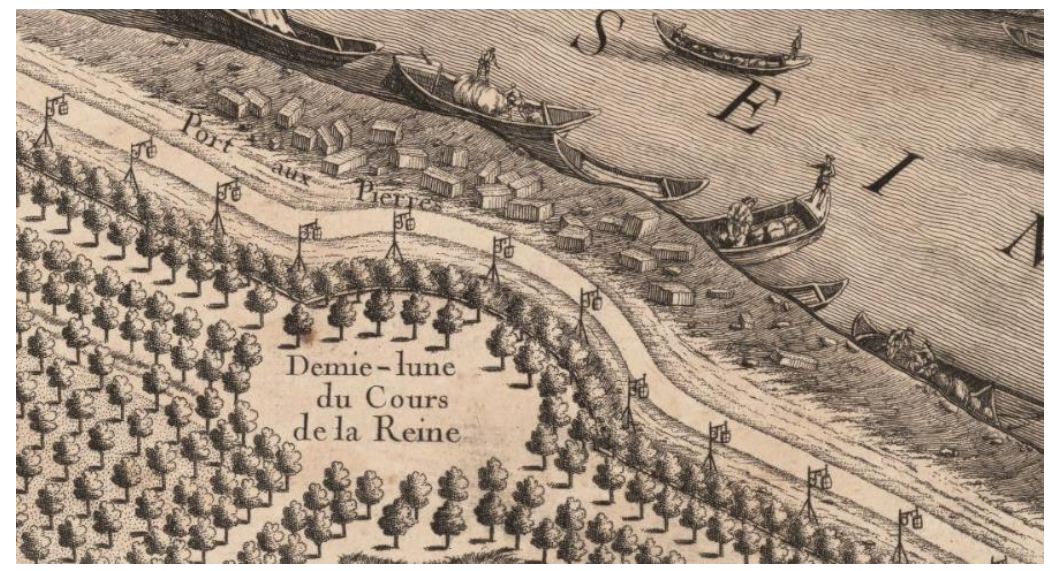

Figure 4. Détail du plan de Paris dit « plan de Turgot » levé et dessiné par Louis Bretez, Paris, 1739

L'exemple du Cours-la-Reine nous apparait comme le lieu idéal pour mettre en place le processus de normalisation spatiale. En tant qu'espace récemment aménagé, c'est un territoire vierge où l'administration peut mettre en valeur un idéal d'ordre urbain par un ordonnancement spatial avec un effort moindre - sans percée, sans alignement de rue et donc sans résistance des habitants et propriétaires. L'ambition de repenser la ville dans son entièreté, contre «un amas de maisons entassées pêle-mêle, sans système, sans économie, sans dessin » [LAU 53] peut s'épanouir dans ce

\footnotetext{
${ }^{13}$ AN, E 2089 arret du 21 décembre 1728, fol 333.
} 
projet d'éclairage. Ici, l'ordonnancement du Cours par la segmentation spatiale des potences de lanternes peut être conçue et matérialisée sur plan avant son installation. Il est d'ailleurs intégré au plan Turgot. De plus, comme l'indique l'édit de 1728, «cette route est la plus passagère du royaume » : cet échantillon de soixante lanternes à réverbères est donc une excellente vitrine de la modernisation urbaine.

Cet échantillon nous permet de ne plus voir les lanternes comme une simple surcouche de mobilier urbain dès qu'elles deviennent de véritables références et des repères du plan d'agencement spatial. A partir des années 1750, les réformateurs urbains souhaitent appliquer le principe d'uniformité générale[GAR 02]. Nous ancrons donc la pratique du nouvel éclairage public dans cette dynamique, comme antidote de la distribution invisible et irrégulière de l'espace urbain. De plus, l'analyse de Michel Foucault des textes réglementaires et des lieux -casernes, manufactures, etc.- montre les moyens et pratiques de l'ordre pour dresser les corps : par réflexivité l'espace réglé devient un instrument des techniques disciplinaires. Ce chainon dans l'argumentation nous permet de progresser dans l'étude de la lanterne policière.

\subsection{Lanterne et action policière de surveillance : connaissance et lisibilité de la ville}

Une des logiques de l'éclairage dans sa démarche sécuritaire est son moyen de surveillance active : il doit assurer une visibilité de l'espace pour pouvoir agir et interpeller les délinquants et criminels. La lanterne est alors pleinement intégrée dans les outils policiers : par sa fonction éclairante, elle assure une connaissance de l'espace nocturne en vue de le maitriser et de faire face à tous les possibles : «connaître c'est contenir » [ROC 09], expression de Daniel Roche qui peut s'appliquer à cet espace et à cette matérialité urbaine nouvellement révélée aux autorités. Vincent Denis montre dans son étude sur la numérotation des maisons de Paris que les projets de Joly de Fleury concernent l'ensemble du territoire urbain et rentrent plus largement dans la dynamique des écrits des années 1720-1730 qui systématisent la nécessité de connaître la ville pour la mettre en ordre [DEN 04]. Dans le cas des lanternes, cette production de savoir est générée par la visibilité et donc la lisibilité que l'éclairage public procure de la temporalité nocturne.

\subsubsection{Matérialisation et allongement du temps de l'action policière: l'effet pré-panoptique des lanternes}

L'étatisation des mécanismes de discipline passe par l'appareil de police. Nous souhaitons nous arrêter sur cette métaphore mécanique de l'institution policière, au prisme de l'implantation matérielle du parc d'éclairage. Selon Foucault, ce pouvoir, pour s'exercer, doit se donner «l'instrument d'une surveillance permanente, exhaustive, omniprésente, capable de tout rendre visible, mais à condition de se rendre elle-même invisible ».

La lanterne matérialise ainsi les nouvelles réflexions sur la nature et l'efficacité des dispositifs de la société disciplinaire. Par sa fonction éclairante, elle structure une nouvelle visibilité dans et de la ville, concept clé des nouvelles stratégies de discipline qui sera définie à la fin du XVIIIe siècle dans la figure architecturale du panoptique (panopticon) de Jeremy Bentham :

«La visibilité est un piège[...]. De là l'effet majeur du panoptique : induire chez le détenu un état conscient et permanent de visibilité qui assure le fonctionnement automatique du pouvoir. Faire que la surveillance soit permanente dans ses effets, même si elle est discontinue dans son action, que les détenus soient pris dans une situation de pouvoir dont ils sont eux-mêmes les porteurs. »

La matérialité du parc d'éclairage permet donc d'automatiser la surveillance policière en suggérant, par la visibilité permanente générée par les lanternes, une possibilité d'être observé et surveillé, au lieu de multiplier les actions visibles de contrôle -rondes du guet, etc. Nous souhaitons nous arrêter ici sur la temporalité de l'action de l'objet technique : l'artefact d'éclairage, en 
condition normale d'utilisation - dose de combustible respectée, système optimal- permet une continuité de la surveillance, suggérée ou effective par la stabilité de l'effet panoptique de la lumière générée. Nous souhaitons rapprocher cette temporalité avec l'idéal de police continuelle et ininterrompue du Plan de travail pour la sûreté de Paris $(1778)^{14}$ rédigé par le Lieutenant général de police Lenoir, qui vise à instituer une « concertation » entre toutes les forces chargées de la police de la ville pendant l'hiver par une meilleure répartition spatiale et temporelle des forces de police mobiles[VID 10]. Ce plan se base principalement sur l'action des inspecteurs qui doivent surveiller l'espace lorsqu'il n'y a pas de patrouille dans la ville, en interaction avec les missions des commissaires, des «patrouilles des gardes françoises et suisses», des «Invalides », des « officiers de police ayant le département militaire », de la «garde de Paris tant à pied qu'à cheval », des officiers de robe courte et de maréchaussée, des employés des fermes, des pompiers, des « inspecteurs et employés à l'illumination » et enfin des « portefalots » ${ }^{15}$.

Le levier matériel de l'illumination publique permet de basculer d'une surveillance effective à une surveillance possible, d'un rapport direct entre individu et pouvoir de contrôle à un rapport indirect intériorisé par l'individu. Nous notons donc que ce tampon matériel rend possible une distanciation à l'usage de la force et rentre ainsi dans les nouvelles dynamiques de la police : ainsi Guillaute, officier de la maréchaussée, défend dans son Mémoire sur la réformation de la Police (1749) le projet d'une police proche des dynamiques de «civilisation»-au sens de Norbert Elias [ELI 85] - par la diffusion de normes peu à peu intégrées par les populations, orientant les comportements des administrés par une autocontrainte croissante. L'incorporation de l'idée de surveillance, d'un regard porté par la lieutenance de police sur l'ensemble, est suggérée par la contrainte -ici visuelle de la matérialité de la lanterne. La dimension pédagogique passe par un rapport visuel et spatial : l'exemple d'école est donné par l'étude de Michel Foucault sur l'exécution de Damiens dans Surveiller et Punir. Ainsi, la lanterne matérialise la mission de prévention de la police, dans le cadre d'une action non réductible à la répression et au pouvoir coercitif [MIL 06] avec un usage plus modéré de la force au profit de la procédure judiciaire et une volonté de pacifier les rapports entre Police et population. L'éclairage comme action préventive accompagne ainsi la mutation de l'action policière -la police devenant pratique administrative [VID 10] - qui diversifie ses activités et prend part à la reconfiguration et à l'aménagement de l'espace urbain.

\subsubsection{Matérialité et machine policière}

Cette décharge du potentiel de surveillance dans l'artefact grâce à l'effet panoptique de l'éclairage permet de générer une économie du pouvoir. L'artefact d'éclairage se substitue ainsi à la figure humaine policière et cette instrumentation de la surveillance est inscrite dans différentes maximes de l'époque telles «après minuit, chaque lanterne vaut un veilleur de nuit » [ $\mathrm{SCH} 93]$ ou dans le poème Les nouvelles lanternes de 1746 de Adrien-Joseph de Valois d'Orville dédié à «l'inventeur du réverbère » l'abbé de Preigney : "ces nouvelles lanternes serviront dans Paris d'armes, de gardes, de barrières $»^{16}$. Dans le poème Plaintes des filous et écumeurs de bourse à nosseigneurs les réverbères ${ }^{17}$, la symbiose avec le corps policier et sa tâche de surveillance est inscrite au moyen de la métaphore du chien de garde mythologique : «ces réverbères, qui n'illuminent que trop bien, [...] de la police, les cerbères, qui ne nous permettent plus rien ». Dans le

\footnotetext{
${ }^{14} \mathrm{BnF}$, Nouvelles acquisitions françaises, 3247, fol. 129.

${ }^{15} \mathrm{BnF}$, Nouvelles acquisitions françaises, 3247, fol. 129.

${ }^{16}$ Le poème est aussi mentionné dans L'Essai historique, critique, philologique, [...] sur les lanternes, op. cit., et dans [FOU 54].

${ }^{17}$ Poème publié à la suite de [FOU 54].
} 
poème post-Concours Les sultanes nocturnes et ambulantes contre nosseigneurs les réverbères ${ }^{18}$ (1769), l'objet technique est non seulement listé au même titre que les autres éléments constitutifs de l'appareil policer (mouchards, falots, guet, etc.) mais une hiérarchie les établit « en tête » de ce système, c'est à dire en première ligne, inscrivant leur rôle clé dans les procédures d'identification et de surveillance :

«Mais pour courir tant de hazards,

Toujours le réverbère en tête,

Et les pousseux et leurs mouchards,

Les falots, espèces de bète

Heurtant, comme loups plus hagards

Ce Guet qui se croit le Dieu Mars. »

Quelle est la part de cette matérialité dans la construction de cet imaginaire de l'appareil sécuritaire policier ? Difficile de répondre tant ce système est à la fois critiqué et admiré : ainsi Louis-Sébastien Mercier, sans son Tableau de Paris, définit l'appareil policier comme une « machine » dont les éléments constitutifs les plus importants sont «les lanternes et réverbères, les différents corps-de-gardes distribués et les falots errants ». Les artefacts d'éclairage - fixes et mobiles- sont donc vues comme des pièces constituantes de cette grande mécanique, où l'instrumentalisation prend le dessus sur l'humain à l'origine des obsessions policières qui alimentent l'imaginaire d'un système qui saurait constamment localiser les individus. Dans tous les cas, cette surcouche matérielle du parc d'éclairage ne fait que renforcer la rhétorique d'une « machine » policière véhiculée par les écrits policiers de la deuxième moitié du XVIII ${ }^{\mathrm{e}}$ s., que ce soit le mémoire de Lemaire de 1770 ou le Plan de travail pour la sûreté de Paris de 1778 rédigé par Lenoir afin d'articuler et de coordonner toutes les forces de police et militaires de ville autour de la figure du lieutenant de police. Cette «machine » dénoncée et admirée par Mercier se construit un imaginaire dans l'articulation par la lieutenance de mode classiques et anciens de police au-delà des dissensions de compétences, de juridictions et de pratiques.

La matérialisation du contrôle policier répond ainsi au manque d'effectif de cette force et aux enjeux d'économie : l'étude sur le guet de Leclerc du Brillet ${ }^{19}$ fait état de barrières inoccupées la nuit durant l'année 1732 faute d'effectifs suffisants et plus largement de difficultés à maintenir le service après deux heures du matin. Face à l'impossibilité de maintenir une occupation policière -ici militaire- constante de la ville, il faut recourir à des stratagèmes, et passer par des techniques de dissuasion en montrant la force policière : Jean Chagniot présente le Guet comme une force souhaitant montrer sa présence le jour, en se concentrant sur les axes commerciaux et ayant une capacité de se projeter rapidement la nuit en segmentant l'effectif en petits groupes [CHA 85]. De même, Lemaire, dans son mémoire rédigé à la demande de Sartine, décrit la stratégie de projection de la garde :

« Ce mouvement continuel de la garde fait le le plus grand effet et le meilleur. Quoiqu'il ne puisse y avoir qu'environ 120 hommes qui soient en marche à la fois, comme ils passent toujours dans les rues principales, [...] il semble qu'ils remplissent tout Paris : on les voit à tous les instants, et leur présence qui en impose, arrête te prévient une infinité de désordres. »

La lanterne participe donc pleinement de la logique disciplinaire mobilisant la visibilité comme outil majeur du contrôle social. En matérialisant les techniques du faire voir, faire savoir et faire

\footnotetext{
${ }^{18}$ Les sultanes nocturnes et ambulantes contre nosseigneurs les réverbères, 1769 cité dans [FOU 54].

${ }^{19}$ BNF, ms fr 21575 fol 312.
} 
croire, elle donne de l'épaisseur à la surveillance suggérée et plus largement à la rhétorique de la surveillance omnisciente dans les écrits policiers. Ainsi, le mémoire Précis des représentations faites à Monseigneur le procureur général (1756) généré par les inspecteurs, présente le lieutenant de Police comme "porté en même temps dans tous les quartiers » [MIL 06] mais également un déploiement d'espions en nombre plus ou moins important, information justement floutée et entretenue afin de faire croire que potentiellement rien ne peut être ignoré de la lieutenance. Ainsi, si notre parc d'éclairage n'est pas un « réseau » technique [MUS 03] [PIC 14] au sens que les lanternes sont des unités autonomes, le jeu du visible et du vu et des techniques pré-panoptiques mobilisées par l'illumination publique font croire à un réseau projeté de surveillance, à un système relié de l'institution policière.

Ce deuxième moment biographique nous a donné les clés pour mettre en valeur un nouveau mode d'existence de la lanterne à réverbères, où notre objet technique gagne en significations en devenant symbole de l'ensemble - « rue » / «ville »- remodelé par l'administration technicienne. Ce prisme, à la perspective artificialiste, nous permet de redonner, à travers la phase de technophanie de l'objet d'éclairage, l'épaisseur sociale et politique de la lanterne publique, soit d'accéder à sa nature et son essence en s'éloignant des seules figures de ses innovateurs.

\section{Conclusion}

En mettant de côté l'inventeur au profit de l'innovation d'éclairage dans cette biographie, nous avons souhaité faire ressurgir la réalité hautement humaine stabilisée dans de la matière ${ }^{20}$ - en l'occurrence dans l'artifice du réflecteur - et la cristallisation des efforts pour rationaliser et conduire les effets de la lumière. La mise en problème du concours d'éclairage donne sens à l'introduction d'un artifice dans le système, unique moyen de pondérer les utilités contraires «clarté » et « économie », utilités tendues entre la tentation de multiplier les sources lumineuses et la recherche de la moindre consommation en combustible

Les deux moments biographiques - l'objet, l'objet et son milieu - nous ont ainsi permis d'opérer un changement d'échelle nécessaire pour montrer que les intentions de mise en ordre de la rue sont à chercher dans le schème interne de l'artefact d'éclairage. Nous avons étudié la résonance de la lanterne dans l'ensemble -et réciproquement- à travers la transposition des discours de mise en ordre des rayons - la rationalisation opérée par l'artifice du réverbère - à une rhétorique d'ordonnancement de la rue par la lanterne, soit un report, par effet-levier, du mythe prométhéen sur la lumière, à la ville idéalisée. Si l'analogie est suivie, il y a un parallèle entre le contrôle du parcours lumineux et la police des mobilités, entre l'action contre le naturel des rayons et le combat contre l'obscurité naturelle de la nuit. Ainsi, par transitivité, nous souhaitons redonner un rôle central à la matérialité de l'artifice «réverbère » dans la rationalisation et normalisation des pratiques de surveillance de l'espace nocturne parisien.

Aussi, si Lorraine Daston s'intéresse principalement à la naissance et à la mort des objets scientifiques dans son recueil Biographies of Scientific Objects [DAS 00], nous avons souhaité interroger plus largement les modes d'existence de notre artefact d'étude comme emblème politique. Ainsi, si la lanterne à réverbères nous a semblé un acteur digne d'une biographie, c'est par son fort potentiel symbolique lui conférant une vie propre dépassant les intentions de ses fabricants. La biographie de la lanterne s'écrit ainsi au fil de sa trajectoire de vie : ce que le l'inventeur inscrit dans le dispositif technique n'est qu'un «script» de compétences toujours passible de «description», selon l'expression de Madeleine Akrich [AKR 91].

\footnotetext{
${ }^{20}$ Pour reprendre le vocabulaire de Simondon.
} 
En conclusion, nous voyons dans cet biographie d'objet technique un argument supplémentaire pour démontrer que la méthode biographique permet de penser le mode d'existence des innovations [CHO 18 ]. Nous avons ainsi créé deux moments dans ce récit biographique (l'objet- l'objet et son milieu) afin de donner à voir les processus de conception et d'innovation de notre objet via sa genèse technique (processus collaboratif, "rationalité créative ») et son mode d'existence lors de son insertion dans son milieu, mettant en valeur sa dimension politique et sociale (policière).

Cette biographie nous a, d'une part, donné les moyens de créér un laboratoire de la lanterne ex situ afin de faire apparaître les efforts inventifs, théoriques et pratiques cristallisés dans l'artifice du réverbère pour conduire les effets de la lumière : cela a éclairé notre compréhension de l'objet tout en militant pour l'incorporation de la culture technique dans l'activité de conception, centrale dans le processus d'innovation [CHO 17 ] [CHO 18 ].

D'autre part, il nous a fallu quitter l'échelle micro du schème technique dans l'objectif de saisir la lanterne dans son milieu, Ce changement d'échelle nous a donné les clés pour saisir la technophanie de la lanterne publique, soit d'apporter une démonstration de l'entrée de l'objet technique dans le monde des significations et son empreinte dans la culture, loin de toute relation d'alliénation, ce que nous présentons comme un argument supplémentaire au développement de la culture technique.

Dans le cadre d'une enquête biographique plus large dépassant le format de cet article, nous montrons comment, depuis l'atelier de l'inventeur jusqu'à son icône dans la caricature populaire, notre objet technique d'étude est investi de valeurs différenciées, de réinterprétation, de détournement par les opérateurs, et de propriétés inédites ${ }^{21}$ [BOT 18].

\section{Bibliographie}

[AKR 06] AKRICH M., CALLON M., LATOUR B.(dir.), Sociologie de la traduction: textes fondateurs, Paris, Mines ParisTech, les Presses, « Sciences sociales », 2006.

[AKR 91] AKRICH M., «L'analyse socio-technique », in VINCK D. (éd.), La Gestion de la recherche , Bruxelles, De Boeck, 1991, p. 339-353.

[APP 86] APPADURAI A. (éd.), The Social Life of Things. Commodities in Cultural Perspective , Cambridge, Cambridge University Press, 1986.

[BEAU 13] BEAUBOIS V., « Design et technophanie » in Cahiers Simondon, n5 (dir.) Jean-Hugues Barthélémy, L'Harmattan, 2013.

[BEN 12] BENSAUDE VINCENT B., Vie d'objets. Critique, Paris, Centre National des Lettres, 2012, p. 588-598.

[BIT 09] BITTERLING D., L'invention du pré carré, Construction de l'espace français sous l'Ancien Régime, Albin Michel, 2009.

[BOT 18] BOTHEREAU B., À la lanterne! Modes d'existence d'un objet banal, entre imaginaire technique et politique. Invention, économie urbaine, publics et circulations du «réverbère», Paris, Barcelone, XVIIIe s., thèse de doctorat sous la codirection de Liliane Hilaire Pérez et Antoni Roca Rosell, EHESS Paris, 2018.

[BOU 94] BOURDIEU P. "L'illusion biographique », in Raisons pratiques sur la théorie de l'action, Paris, Seuil, 1994, p. 81-89.

[CAR 09] CARBONNIER Y., "La monarchie et l'urbanisme parisien au siècle des Lumières. Grands projets et faiblesse du pouvoir », Histoire urbaine, 2009 (n 24), p. 33-46.

[CHA 85] CHAGNIOT J., Paris et l'armée au XVIIIe siècle. Étude politique et sociale, éd Economica, 1985.

\footnotetext{
${ }^{21}$ Pour une étude du mode d'existence de la lanterne politique révolutionnaire voir Benjamin Bothereau, À la lanterne ! Modes d'existence d'un objet banal, entre imaginaire technique et politique. Invention, économie urbaine, publics et circulations du "réverbère», Paris, Barcelone, XVIIle s., op.cit.
} 
[CHO 17] CHOUTEAU M., FOREST J., NGUYEN C., Technologie et innovation, Numéro : Culture Technique et Culture d'Innovation, 2017.

[CHO 18] CHOUTEAU M., FOREST J., NGUYEN C., Genèse des innovations. Les biographies comme vecteur de connaissances du processus de l'innovations, Editions de l'UTBM, 2018.

[CUV 15] CUVELIER L., « Rendre visible l'autorité. Politiques de l'information et communication des représentants en mission à l'armée des Pyrénées orientales », Annales historiques de la Révolution française, 382, 2015, 31-6.

[DAS 00] DASTON L. (éd.), Biographies of Scientific Objects, Chicago, The University of Chicago Press, 2000.

[DEN 04] DENIS V., MILLIOT V., «Police et identification dans la France des Lumières », Genèses, vol. n54, no. 1, 2004, pp. 4-27.

[DES 97] DESPORTES M., PICON A., De l'espace au territoire, L'aménagement en France XVIe-XXe siècle, Presses de l'ENPC, 1997.

[DUB 08] DUBOURG GLATIGNY P., VERIN H. (dir.), Réduire en art. La technologie de la Renaissance aux Lumières, Paris, Éditions de la Maison des Sciences de l'Homme, 2008.

[ELI 85] ELIAS N., La société de cour, Paris, Flammarion, 1985.

[FOU 54] Fournier E., Les Lanternes. Histoire de l'ancien éclairage de Paris, Paris, 1854

[FOU 75] FOUCAULT M., Surveiller et Punir, naissance de la prison, Paris, Gallimard, 1975.

[GAR 02] GARRIOCH D., The Making of Revolutionary Paris, Berkeley, Los Angeles, Londres, University of California Press, 2002.

[GAR 03] GARÇON A.F., « Comment retracer historiquement les chemins de la novation », In L. HILAIRE-PEREZ, A.F. GARÇON (dir.), Les chemins de la nouveauté. Innover, inventer au regard de l'histoire, éditions du CTHS, Paris, p. 443-470, 2003.

[LAT 91] LATOUR B., Nous n'avons jamais été modernes, Essai d'anthropologie symétrique, Paris, La Découverte, 1991.

[MIL 06] MILLIOT V.(dir.), Les Mémoires policiers, 1750-1850. Écritures et pratiques policières du Siècle des Lumières au Second Empire, Presses universitaires de Rennes, 2006.

[MAR 03] MARIN B., "Administrations policières, réformes et découpages territoriaux (XVIIe-XIXe siècle », MEFRIM, 115, 2003.

[MUS 03] MUSSO P., (dir.), Réseaux et société, Paris, Presses universitaires de France, coll. La politique éclatée, 2003.

[MUS 15] MUSSO P., L’imaginaire industriel, Paris, édition Manucius, 2015.

[NAP 03] NAPOLI P., Naissance de la police moderne, Pouvoir, normes, société, Paris, La Découverte, 2003.

[PAT 69] Patte P., Mémoire sur les objets les plus importants de l'architecture, Paris, Rozet, 1769.

[PIC 03] PICON A., La Ville des réseaux : Un imaginaire politique, Editions Manucius, 2014.

[POT 09a] Potofsky A., Constructing Paris in the Age of Revolution, New York, Palgrave Macmillan, 2009.

[POT 09b] POTOFSKY A., «L'État révolutionnaire et les corporations du bâtiment à Paris, 1789-1792 », Histoire Urbaine, 1(24), 2009.

[REV 96] REVEL J. (dir.), Jeux échelles La micro-analyse à l'expérience, Paris, Gallimard-Le Seuil, Hautes études, 1996.

[ROC 09] ROCHE D., «Mémoires policiers, une culture de corps » in DENYS C., MARIN B., MILLIOT V., Réformer la police. Les mémoires policiers en Europe au XVIIIe siècle, Presses Universitaires de Rennes 2009.

[SCH 93] SCHIVELBUSCH W., La nuit désenchantée, Paris, Gallimard, 1993.

[SIM 60] SIMONDON G., "Psychologie de la technicité », Bulletin de l'Ecole pratique de psychologie et de pédagogie de Lyon, 1960.

[SIM 89] SIMONDON G., Du mode d'existence des objets techniques, (1958) Paris, Aubier, 1989.

[VER 05] VERBEEK P., What Things Do. Philosophical Reflections on Technology, Agency and Design , University Park, Pennsylvania State University Press, 2005. 
[VER 93] VÉRIN H., La gloire des ingénieurs. L'intelligence technique du XVI ${ }^{\mathrm{e}}$ au $\mathrm{XVIII}^{\mathrm{e}}$ siècle, Paris, Albin Michel, 1993.

[VIE 74] Vieil (Le) P., L'art de la peinture sur verre et de la vitrerie, 1774.

[VID 10] VIDONI N., «Les « officiers de police » à Paris (milieu XVIIe-XVIIIe siècle) », Rives méditerranéennes, 2010. 